\title{
An interview with Heon Ju Lee on ROCKIT Healthcare's novel bioprinting treatment for dermal scarring
}

\author{
Heon Ju Lee*,1 \\ ${ }^{1}$ Rokit Healthcare, B-1106, Gabul Great Valley, 32 Digital-ro 9-gil, Geumcheon-gu, Seoul, South Korea \\ *Author for correspondence: heonjulee@yu.ac.kr
}

In this exclusive interview, Heon Ju Lee discusses ROCKIT healthcare's novel bioprinting technique used to treat patients with dermal scarring. This interview was conducted by Mike Gregg, Commissioning Editor of the Journal of 3D Printing in Medicine.

Dr Heon Ju Lee is the Chief Technology Officer and Managing Director of ROKIT. He is developing the service platform technology for artificial organ regeneration and supervises the overseas business development for the propagation of such service platforms. The focus of these platforms, bringing bio 3D print-based medical therapies into the operating room, on tissues that are relatively easy to fabricate structurally with the current technology, this includes skin, cartilage, hair, retina and heart patch regeneration. Dr Lee has a PhD from MIT in mechanical engineering and has been working as a 3D/4D printing group leader at KIST.

First draft submitted: 15 April 2019; Accepted for publication: 17 April 2019; Published online: 21 August 2019

Keywords: autologous cells $\bullet$ biofabrication $\bullet$ bioprinting $\bullet$ dermal scarring $\bullet$ tissue regeneration

\section{Could you please briefly describe your current research focus? What led you to this area of} study?

We are focusing on the development of a tissue regeneration therapeutic platform utilizing autologous tissue/cells and proprietary three-dimensional bioprinting technology.

With the general increase in life expectancy across the globe and the increasing scarcity of financial resources dedicated toward centralized healthcare systems, the need for innovative, personalized medicine is higher than ever. Our company has sought ways to not only improve the safety and efficacy of current therapies but also to reduce costs borne by the patients, and has identified regenerative medicine as an area with great potential, especially because we can apply our techniques in the clinic right away if we operate within the 'autologous realm.' Bringing three-dimensional bioprinting technology to the clinic reduces costs by cutting down the transactional costs associated with logistics of drug manufacturing and tissue processing.

You have recently been working on the development of a novel dermal patch graft using 3D biofabrication technology. Could you please tell me a bit more about the technology used \& how the patch grafts are made?

For tissue regeneration, three-dimensional structures with the same physicochemical properties as the target tissue or organ need to be formed using multiple elements that include stem cells, growth factors, adult cells and extracellular matrices.

First, stem cells are an essential element to restore damaged tissues in a short period of time due to their great regenerative capability. The stem cells receive signals via growth factors, extracellular matrix and the adult cells of the target tissues which guide them to proliferate and differentiate into the desired tissue cells. In addition, the materials in a graft must be homogeneously mixed in the form of a scaffold that recapitulates the native tissue characteristics as well as maintaining a specific shape to fit into the damaged site.

Future Medicine 
The autologous dermal patch graft we developed has mechanical properties similar to skin tissue and includes the aforementioned stem cells, growth factors and extracellular matrix. It is produced using our 3D bioprinter INVIVO at a uniform cell concentration, accurate size and thickness to the debrided skin wound site. The primary purpose of using a $3 \mathrm{D}$ bio-printer is to maintain uniform cell density to minimize cell death or overgrowth due to local necrosis. In addition, it is necessary to be able to produce skin patches with constant quality every time it is printed. In particular, ROKIT healthcare 3D bioprinter INVIVO can safely protect cells from contamination and increases cell viability by using the sterilization function built into the device.

\section{How do you collect the autologous stem cells from the patient to produce the bioink?}

The safest method to obtain fat tissue is through liposuction to acquire the patient's own stem cells and extracellular matrix. To isolate the stem cells and extracellular matrix we used a technique called stromal vascular fraction (SVF) isolation.

The acquired SVF cells and extracellular matrix were then mixed with a medically approved hydrogel chosen due to its similar mechanical properties as human skin tissue to produce a 'bio-ink' which then can be printed using INVIVO to generate the dermal patch graft.

\section{How do you ensure that the INVIVO 3D Bioprinter prints the autologous stem cells uniformly in the dermal patch grafts? \\ By keeping the viscosity of the hydrogel constant, we ensured that the stem cells dispersed uniformly in the bio-ink during printing. We also used a bioprinting technique to print out or locate cells in a constant spatial arrangement.}

What are some of the challenges \& current limitations associated with this technology $\&$ the production of the dermal patch graft? What needs to be done?

As of now, because our ability to harvest the autologous adipose tissue is limited by the amount of fat that is liposuctioned from the patient, we are only able to print skin patches that are a maximum of $10 \mathrm{~cm} \times 10 \mathrm{~cm}$. This is a disadvantage as several patches need to be printed in order to apply to an area with large dermis damage.

\section{What are the intended clinical applications of these dermal patch grafts? How could this work benefit patients in the future?}

Our intended clinical application includes dermatological wounds and scars due to burns, diabetic foot ulcers, pressure ulcers, etc. which have no other treatment option besides full-thickness skin transplantation. Of course, our technology can also be applied to the removal of skin scars or tattoos for surgical procedures pertaining to beauty care and cosmetics.

What future plans do you have for this technology to be evaluated in clinical studies? Could you tell me a bit more about this?

Starting with scar revision, we plan to expand treatment to burns, diabetic foot ulcer and pressure ulcer.

Are you currently studying the potential application of this biofabrication technology for any other regenerative therapeutic strategies?

Currently, we are also developing applications such as cartilage regeneration for degenerative arthritis, retinal regeneration for macular degeneration retinal damage, myocardial patch for regeneration of myocardial tissue due to myocardial infarction. For cartilage regeneration, we plan to start clinical research in near future based on the successful completion of hyaline cartilage regeneration in large animal studies.

What obstacles do you expect you will need to overcome in order to transfer your technique to treating conditions such as burns, pressure ulcers \& diabetic foot ulcers?

The operation time can be prolonged for application to a wide burn damaged area, and in the case of urethra or pressure ulcers, this greatly decreases the immunity as well as the regeneration capability of the patient. Therefore, skin patches can and should be applied with other fundamental treatments to boost overall patient's health in order to achieve the expected dermis regeneration. 
How do you envision 3D printing technologies evolving in your field? How do you think this may help to further develop your technique for medical applications of the future?

Two of the biggest advantages of general 3D printing are: you can create your own custom design, and you can make them anywhere the material (bioink) is available. We expect the medical healthcare system to move toward increasing polarization, where on one end sits standardized reimbursed therapies, and on the other high-premium, personalized, nonreimbursed therapies. 3D bioprinting techniques can play a crucial role when targeting the personalized premium market, and that is exactly where we see opportunities. Introduction of the idea of 'bedside manufacturing' using 3D bioprinters will greatly impact the medicoeconomic aspects by reducing logistics and manufacturing costs associated with medical supply, which can lead to much improved management of public healthcare.

Financial \& competing interests disclosure

HJ Lee has no relevant affiliations or financial involvement with any organization or entity with a financial interest in or financial conflict with the subject matter or materials discussed in the manuscript. This includes employment, consultancies, honoraria, stock ownership or options, expert testimony, grants or patents received or pending, or royalties.

No writing assistance was utilized in the production of this manuscript.

\section{Disclaimer}

The opinions expressed in this interview are those of $\mathrm{HJ}$ Lee and do not necessarily reflect the views of Future Medicine Ltd. 
